\title{
Trauma em idosos socorridos pelo serviço de atendimento móvel de urgência
}

\author{
Trauma in elderly people assisted by the mobile emergency care service
}

Lesiones en adultos mayores socorridos por el servicio de atención móvil de urgencia

Danielle Braga Pena Franck ${ }^{1}$ ib https://orcid.org/0000-0003-2473-0481

Yule Caroline Nunes da Costa ${ }^{1}$ ic https://orcid.org/0000-0003-0975-9411

Katiusse Rezende Alves ${ }^{2}$ io https://orcid.org/0000-0003-3393-8567

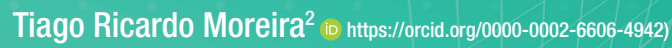

Nádia Fontoura Sanhudo ${ }^{1}$ io hitps://orcid.orgy/0000-0001-9714-2854

Geovana Brandão Santana Almeida' ${ }^{1}$ is https://orcid.org/0000-0003-3865-9727

Vilanice Alves de Araújo Püschel ${ }^{3}$ io https://orcid.org/0000-0001-6375-3876

Fábio da Costa Carbogim ${ }^{1}$ io https://orcid.org/0000-0003-2065-5998

Como citar:

Franck DB, Costa YC, Alves KR, Moreira TR,

Sanhudo NF, Almeida GB, et al. Trauma em

idosos socorridos pelo serviço de atendimento móvel de urgência. Acta Paul Enferm. 2021;34:eAPE03081.

DOI

http://dx.doi.org/10.37689/actaape/2021A003081

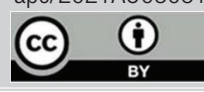

Enfermagem geriátrica; Idoso; Serviços médicos de emergência; Ferimentos e lesões

Keywords

Geriatric nursing; Aged; Emergency medical services; Wounds and injuries

Descriptores

Enfermería geriátrica; Anciano; Servicios médicos de urgência; Heridas y traumatismos

\section{Submetido \\ 21 de Novembro de 2019 \\ Aceito \\ 1 de Junho de 2020}

Autor correspondente

Fábio da Costa Carbogim E-mail: fabio.carbogim@ufjf.edu.br

\section{Resumo}

Objetivo: Descrever as ocorrências e os mecanismos de trauma em idosos socorridos pelo Serviço de Atendimento Móvel de Urgência.

Métodos: Estudo transversal, realizado com registros de atendimento de trauma em idosos, em cidade de porte médio, com população estimada de 600 mil habitantes. Foram analisadas as variáveis: faixa etária, sexo, ano, mecanismo de trauma, região geográfica do atendimento e óbito. Os dados foram analisados por meio de frequências absolutas e relativas e, quando apropriado, por médias e desvio padrão. As diferenças estatísticas foram avaliadas pelo teste qui-quadrado, para variáveis categóricas, e ANOVA, para contínuas.

Resultado: A maioria dos envolvidos era do sexo feminino, com destaque para eventos de quedas. Os idosos com faixa etária entre 60 e 69 anos foram mais expostos à violência no trânsito e à violência interpessoal; já os idosos entre 70 e 89 anos, a quedas. A forma mais comum de trauma foi queda de altura (17,5\%), da própria altura (62,3\%), seguida da violência no trânsito e acidentes (12,7\%). A região da cidade com maiores registros de atendimento por quedas, violência interpessoal e no trânsito foi a área central. 0 óbito foi 0 desfecho mais frequente entre homens $(p<0,001)$ e entre pessoas que sofreram quedas $(p=0,024)$.

Conclusão: Os resultados podem contribuir para a compreensão dos mecanismos de trauma no idoso e 0 direcionamento de políticas de saúde no âmbito da promoção, da prevenção e da recuperação da saúde.

\section{Abstract}

Objective: To describe trauma occurrences and mechanisms in elderly people assisted by the Mobile Emergency Care Service (SAMU).

Methods: Cross-sectional study conducted with records of trauma care in elderly subjects in a medium-sized city with an estimated population of 600 thousand inhabitants. The following variables were analyzed: age group, sex, year, trauma mechanism, geographic region of care and death. Data were analyzed by means of absolute and relative frequencies and when appropriate, by means and standard deviation. Statistical differences were assessed using the chi-square test for categorical variables and the ANOVA for continuous variables.

Result: Most people involved were female, with emphasis on falls events. Elderly people aged 60-69 years were more exposed to traffic violence and interpersonal violence, while those aged 70-89 years were more exposed to falls. The most common form of trauma was fall from height (17.5\%) and fall from one's own height $(62.3 \%)$, followed by traffic violence and accidents $(12.7 \%)$. The central region of the city had the highest number of records of care due to falls, interpersonal violence and traffic violence. Death was the most frequent outcome among men $(p<0.001)$ and among people who suffered falls $(p=0.024)$.

Universidade Federal de Juiz de Fora, Juiz de Fora, MG, Brasil.

2Universidade Federal de Viçosa, Viçosa, MG, Brasil.

${ }^{3}$ Escola de Enfermagem da Universidade de São Paulo, São Paulo, SP, Brasil.

Conflitos de interesse: nada a declarar. 
Conclusion: The results can contribute to understand trauma mechanisms in elderly people and direct health policies within the scope of health promotion, prevention and recovery.

\section{Resumen}

Objetivo: Describir los casos y los mecanismos de lesión en adultos mayores socorridos por el Servicio de Atención Móvil de Urgencia.

Métodos: Estudio transversal, realizado con registros de atención de lesiones por trauma en adultos mayores, en una ciudad de tamaño medio, con una población estimada de 600.000 habitantes. Las variables analizadas fueron: grupo de edad, sexo, año, mecanismo de lesión, región geográfica de la atención y fallecimiento. Los datos fueron analizados mediante frecuencias absolutas y relativas y, cuando fue conveniente, por promedios y desviación típica. Las diferencias estadísticas fueron analizadas con la prueba $\chi^{2}$ de Pearson en variables categóricas y con ANOVA en variables continuas.

Resultado: La mayoría de las personas involucradas era de sexo femenino, con énfasis en eventos de caídas. Los adultos mayores del grupo de edad entre 60 y 69 años estuvieron más expuestos a la violencia en el tránsito y a la violencia interpersonal y los del grupo entre 70 y 89 años a caídas. La forma más común de lesión fue caída de altura (17,5\%), de la propia altura (62,3 \%), seguida por la violencia en el tránsito y accidentes (12,7 \%). La región de la ciudad con mayores registros de atención por caídas, violencia interpersonal y en el tránsito fue el área central. El fallecimiento fue el desenlace más frecuente entre los hombres $(p<0,001)$ y entre las personas que sufrieron caídas $(p=0,024)$.

Conclusión: Los resultados pueden contribuir para comprender los mecanismos de lesión en el adulto mayor y orientar políticas sanitarias en el ámbito de la promoción, prevención y recuperación de la salud.

\section{Introdução}

A população idosa brasileira atingiu a marca de 30 milhões no ano de 2019, o que tem levado especialistas a estimar que, no ano de 2030, o país será a quinta maior população idosa mundial. ${ }^{(1,2)}$ Paralelamente, com o avanço das políticas de saúde e o estímulo a práticas e hábitos de vida saudáveis, essa população tem vivido de forma ativa, retardando o aparecimento de défices orgânicos naturais ao envelhecimento. ${ }^{(2)}$ Dessa forma, mais importante que estabelecer o início da faixa etária que define uma pessoa como idosa é mapear e definir as vulnerabilidades comuns a essa população. ${ }^{(2,3)}$

Estudos apontam que fatores intrínsecos ao envelhecimento, como défices motores, sensoriais e cognitivos, são fragilidades que podem aparecer de forma sutil ao longo do tempo, expondo os idosos a maior risco de trauma e complicações. ${ }^{(3,4)}$ Estes, somados aos fatores extrínsecos, como má iluminação, pisos escorregadios e irregulares, presença de obstáculos, condiçóes das vias públicas, entre outros, contribuem sobremaneira para o desencadeamento do trauma. ${ }^{(3-6)}$

O trauma caracteriza-se por um conjunto de perturbaçóes lesivas provocadas por um agente físico, com extensão e etiologias diversificadas. ${ }^{(7,8)}$ É a terceira maior causa de óbito no país, atrás somente das doenças cardiovasculares e das neoplasias malignas. ${ }^{(4)}$ Investigaçóes destacam que a causa mais comum de trauma na população idosa são as quedas, seguidas pelos acidentes de trânsito e violência. ${ }^{(4,8)}$
Para essa população, as quedas representam até dois terços dos acidentes, o que a torna um dos principais previsores de morbimortalidade no país. ${ }^{(3,8)}$ No ano de 2013, foram registradas 93.312 internaçóes por quedas em pessoas acima de 60 anos, o que representou aproximadamente 256 idosos internados por queda ao dia. ${ }^{(4,9)}$

Náo raro, o trauma em idosos resulta em lesóes e fraturas que comprometem as Atividades da Vida Diária, elevam os índices de institucionalização e podem progredir para declínio do estado geral de saúde. ${ }^{(7)}$ Isso, por sua vez, avoluma os custos com hospitalização e outros serviços de saúde, mostrando-se, dessa maneira, como importante problema para a saúde pública e a assistência de enfermagem. ${ }^{(3,8-10)}$

Um estudo de séries temporais analisou a magnitude e a tendência das taxas de internação por lesôes traumáticas em unidades de terapia intensiva em 18 anos. Os resultados demonstraram que o trauma representou a quarta causa de internaçóes em unidades de terapia intensiva. As taxas foram duas vezes mais elevadas em homens e com crescimento médio anual maior para pessoas de 60 anos ou mais. ${ }^{(11)}$

Para a equipe multiprofissional, na qual insere-se a enfermagem, estudos epidemiológicos sobre trauma em idosos podem contribuir com informaçôes para planejamento, estratégias assistenciais e educação continuada profissional. Em geral, a equipe de enfermagem é a primeira a prestar o atendimento ao paciente idoso (poli)traumatizado; logo, políticas 
de qualificação, baseadas em evidências científicas, podem contribuir para a redução de complicaçôes e sequelas temporárias ou permanentes. ${ }^{(5,6,8)}$

Por outro lado, apesar da crescente elevação da ocorrência de trauma na populaçáo idosa, a literatura ainda carece de investigaçóes que identifiquem os fatores de risco, mecanismos de trauma, faixa etária, sexo, entre outras variáveis que contribuam para prevenção e direcionamento das políticas públicas. ${ }^{(8,10,11)}$

Diante do exposto e da importância de investigaçóes que avaliem e estudem o impacto da vulnerabilidade da populaçáo idosa, de acordo com o estabelecido pela Agenda Nacional de Prioridades de Pesquisa em Saúde, ${ }^{(12)}$ justifica-se a presente pesquisa, que teve por objetivo descrever as ocorrências e os mecanismos de trauma em idosos socorridos pelo Serviço de Atendimento Móvel de Urgência (SAMU).

\section{Métodos}

Trata-se de estudo transversal, desenvolvido a partir de registros do SAMU em cidade de porte médio no interior de Minas Gerais, com população estimada de 600 mil habitantes. Foram incluídos todos os registros de trauma em pessoas com faixa etária superior a 60 anos, entre os anos de 2014 e 2018. Do total de 6.233 registros, 193 (3\%) foram excluídos por estarem incompletos ou duplicados.

$\mathrm{O}$ ano de 2014 foi estabelecido como marco para início da coleta de dados, pois o SAMU se vinculou ao Consórcio Intermunicipal de Saúde da Região Sudeste, informatizando todos os registros, o que passou a garantir padronização nas informaçóes, com dados colhidos em tempo real.

$\mathrm{O}$ início do preenchimento das informaçóes ocorre a partir do acionamento do SAMU pela população, com dados como nome, faixa etária, sexo, local da ocorrência e motivo da solicitação. Durante o atendimento, a equipe informa o quadro clínico da vítima, via dispositivo eletrônico com acesso à internet ou telefone do serviço de urgência (telefone 192), para o regulador, que orienta os procedimentos que devem ser realizados e para qual unidade hospitalar a vítima deve ser encaminhada.
Posteriormente, com a finalização do atendimento pelo SAMU, todas essas informaçôes são armazenadas em banco de dados.

O roteiro de coleta de dados e a organização das informações formam realizados pelos pesquisadores, seguindo as informaçóes contidas no banco de dados do SAMU. Estabeleceu-se como variável dependente o trauma e, como variáveis independentes, idade, sexo, ano, mecanismo de trauma (tipo de trauma), região geográfica do atendimento e óbito.

Foi feita análise descritiva das variáveis sexo, faixa etária, região geográfica, mecanismo de trauma e ocorrência de óbito em relaçáo aos anos de 2014 a 2018. A seguir, avaliaram-se sexo, faixa etária e região geográfica em relação aos principais mecanismos de trauma, e, posteriormente, a ocorrência de óbito foi descrita em relação a sexo, faixa etária, região geográfica, mecanismo de trauma e ano.

$\mathrm{Na}$ análise de dados, cabe destacar que os principais mecanismos causadores de trauma foram agrupados em quatro categorias: quedas (queda da própria altura, de altura, de bicicleta, de cavalo e de moto), violência interpessoal (agressão física, autoagressão, ferimento com arma branca e ferimento com arma de fogo), violência de trânsito (acidentes envolvendo carro/moto/bicicleta e atropelamento) e traumas genéricos (acidente trabalho e com máquina agrícola, afogamento, agressão por animal, ferimento superficial qualquer, queimadura, trauma elétrico e trauma musculoesquelético isolado). Foi feita a opção por nominar de "traumas genéricos" os mecanismos lesivos menos frequentes. A ocorrência de óbito foi categorizada em presente ou ausente.

A variável idade foi categorizada nas faixas etárias 60 a 69 anos, 70 a 79 anos, 80 a 89 anos e maior ou igual a 90 anos. A variável região do município em que ocorreu o trauma foi organizada em central, norte, nordeste, sul, sudeste, leste, oeste e rural.

As variáveis categóricas foram apresentadas por meio de frequências absolutas e relativas, e as contínuas, por médias e desvio padrão. Para as variáveis categóricas, quando apropriado, as diferenças estatísticas foram avaliadas pelo teste qui-quadrado ou exato de Fisher. Para as variáveis contínuas, foi aplicada a Análise da Variância (ANOVA), quando apropriado. 
Tabela 1. Caracterização de atendimentos pré-hospitalares a idosos vítimas de trauma entre 2014 e 2018 ( $n=6040)$

\begin{tabular}{|c|c|c|c|c|c|c|c|c|}
\hline Variáveis & & $\begin{array}{l}2014 \\
\mathrm{n}(\%)\end{array}$ & $\begin{array}{l}2015 \\
\mathrm{n}(\%)\end{array}$ & $\begin{array}{l}2016 \\
\mathrm{n}(\%)\end{array}$ & $\begin{array}{l}2017 \\
\mathrm{n}(\%)\end{array}$ & $\begin{array}{l}2018 \\
\mathrm{n}(\%)\end{array}$ & $\begin{array}{l}\text { Total } \\
\mathrm{n}(\%)\end{array}$ & p-value $e^{\ddagger}$ \\
\hline \multirow[t]{3}{*}{ Sexo $0^{\circ}$} & Feminino & $475(59,5)$ & $630(56,7)$ & $688(53,1)$ & $720(56,4)$ & $860(55,1)$ & $3373(55,8)$ & 0,059 \\
\hline & Masculino & $323(40,5)$ & $481(43,3)$ & $607(46,9)$ & $556(43,6)$ & $700(44,9)$ & $2667(44,2)$ & \\
\hline & & $76,3(9,6)$ & $75,6(9,4)$ & $75,6(9,6)$ & $75,8(9,7)$ & $74,6(10,3)$ & $75,5(9,8)$ & $0,007^{\S}$ \\
\hline \multirow[t]{4}{*}{ Faixa etária ${ }^{+}$} & $60-69$ & $226(28,3)$ & $330(29,7)$ & $418(32,3)$ & $410(32,1)$ & $576(36,9)$ & $1960(32,4)$ & 0,006 \\
\hline & $70-79$ & $255(32,0)$ & $360(32,4)$ & $394(30,4)$ & $384(30,1)$ & $441(28,3)$ & $1834(30,4)$ & \\
\hline & 80-89 & $236(29,6)$ & $327(29,4)$ & $361(27,9)$ & $365(28,6)$ & $400(25,6)$ & $1689(28,0)$ & \\
\hline & $\geq 90$ anos & $81(10,1)$ & $94(8,5)$ & $122(9,4)$ & $117(9,2)$ & $143(9,2)$ & $557(9,2)$ & \\
\hline \multirow[t]{6}{*}{ Região geográfica* } & Central & $256(32,0)$ & $375(33,7)$ & $419(32,4)$ & $427(33,5)$ & $479(30,7)$ & $1956(32,4)$ & $<0,001$ \\
\hline & Leste & $109(13,7)$ & $211(19,0)$ & $237(18,3)$ & $174(13,6)$ & $242(15,5)$ & $973(16,1)$ & \\
\hline & Norte & $220(27,6)$ & $291(26,2)$ & $343(26,5)$ & $355(27,8)$ & $542(34,7)$ & $1751(29,0)$ & \\
\hline & Oeste & $48(6,0)$ & $64(5,8)$ & $62(4,8)$ & $78(6,2)$ & $75(4,8)$ & $327(5,4)$ & \\
\hline & Sul & $121(15,2)$ & $155(13,9)$ & $197(15,2)$ & $211(16,5)$ & $202(13,0)$ & $886(14,7)$ & \\
\hline & Rural & $44(5,5)$ & $1(1,4)$ & $37(2,8)$ & $31(2,4)$ & $20(1,3)$ & $147(2,4)$ & \\
\hline \multirow[t]{4}{*}{ Tipo de trauma* } & $A G$ & $29(3,6)$ & $58(5,2)$ & $71(5,5)$ & $80(6,3)$ & $59(6,3)$ & $297(4,9)$ & $<0,006$ \\
\hline & Quedas & $652(81,7)$ & $911(82,0)$ & $1039(80,2)$ & $1019(79,9)$ & $1259(80,8)$ & $4880(80,8)$ & \\
\hline & $\mathrm{VI}$ & $28(3,5)$ & $15(1,4)$ & $28(2,2)$ & $21(1,6)$ & $34(2,2)$ & $126(2,1)$ & \\
\hline & VT & $89(11,2)$ & $127(11,4)$ & $157(12,1)$ & $156(12,2)$ & $208(13,3)$ & $737(12,2)$ & \\
\hline \multirow[t]{2}{*}{ Óbito* } & Não & $775(97,1)$ & $1087(97,8)$ & $1275(98,5)$ & $1264(99,0)$ & $1526(97,8)$ & $5927(98,1)$ & 0,016 \\
\hline & Sim & $23(2,9)$ & $24(2,2)$ & $20(1,6)$ & $12(1,0)$ & $34(2,2)$ & $113(1,9)$ & \\
\hline
\end{tabular}

Fonte: Serviço de Atendimento Móvel de Urgência de Juiz de Fora, Minas Gerais.

† teste qui-quadrado; " Frequência absoluta e relativa; † Média e desvio-padrão; ${ }^{\S}$ Anova; AG - Acidente genérico; VI - Violência interpessoal; VT - Violência no trânsito

As análises foram realizadas no Statistical Software Stata/SE Version 13.1 (Stata-Corp, College Station, Texas), a um nível de confiança de $95 \%(\mathrm{p}<0,05)$.

A pesquisa foi iniciada após aprovação do Comitê de Ética em Pesquisa com Seres Humanos, sob Certificado de Apresentação para Apreciação Ética (CAAE) 83369818.3.0000.5147 e parecer 2.586 .021 .

\section{Resultados}

Fizeram parte do estudo 6.040 registros. A maioria dos atendimentos foi prestado a idosos do sexo feminino $(55,8 \%)$, com média de 75,5 anos (desvio padrão: 9,8$)$, que sofreram quedas $(80,8 \%)$ na regiáo central da cidade $(32,4 \%)$. Do total de registros, $113(1,9 \%)$ idosos evoluíram para óbito (Tabela 1).

Quanto aos mecanismos de trauma, os mais comuns foram as quedas de altura $(17,5 \%)$ e da própria altura $(62,3 \%)$, seguidas da violência no trânsito, sobretudo atropelamentos $(6,1 \%)$ e acidentes envolvendo carro, moto e bicicleta (6,6\%). Para mecanismos genéricos, 297 (4,9\%) registros definiram-se como outras formas de trauma, além das quedas, violência de trânsito e violência interpessoal (Tabela 2).
Tabela 2. Mecanismos de trauma em idosos e desfechos registrados pelo Serviço de Atendimento Móvel de Urgência entre 2014e $2018(n=6040)$

\begin{tabular}{|c|c|c|c|}
\hline \multirow[b]{2}{*}{ Mecanismos de trauma } & \multicolumn{3}{|c|}{ Ocorrência de óbito } \\
\hline & $\begin{array}{c}\text { Não } \\
(\mathrm{n}=5927) \\
\mathrm{n}(\%)\end{array}$ & $\begin{array}{c}\underset{\text { Sim }}{(n=113)} \\
n(\%)\end{array}$ & $\begin{array}{c}\text { Total } \\
(\mathrm{n}=6040) \\
\mathrm{n}(\%)\end{array}$ \\
\hline Acidente trabalho/máquina agrícola* & $15(0,2)$ & $0(0,0)$ & $15(0,2)$ \\
\hline Acidente envolvendo carro/moto/bicicleta & $391(6,6)$ & $10(8,8)$ & $401(6,6)$ \\
\hline Afogamento* & $4(0,1)$ & $1(0,8)$ & $5(0,1)$ \\
\hline Agressão animal ${ }^{*}$ & $19(0,3)$ & $0(0,0)$ & $19(0,3)$ \\
\hline Agressão física & $94(1,6)$ & $2(1,8)$ & $96(1,6)$ \\
\hline Atropelamento & $356(6,0)$ & $14(12,4)$ & $370(6,1)$ \\
\hline Ferimento de arma branca & $19(0,3)$ & $0(0,0)$ & $19(0,3)$ \\
\hline Ferimento de arma de fogo & $9(0,1)$ & $2(1,8)$ & $11(0,2)$ \\
\hline Ferimento superficial qualquer ${ }^{*}$ & $88(1,5)$ & $0(0,0)$ & $88(1,5)$ \\
\hline Queda da própria altura & $3704(62,5)$ & $56(49,6)$ & $3760(62,3)$ \\
\hline Queda de altura & $1029(17,4)$ & $26(23,0)$ & $1055(17,5)$ \\
\hline Queda de bicicleta & $28(0,5)$ & $0(0,0)$ & $28(0,5)$ \\
\hline Queda de cavalo & $3(0,1)$ & $0(0,0)$ & $3(0,1)$ \\
\hline Queimadura* & $15(0,2)$ & $0(0,0)$ & $15(0,2)$ \\
\hline Trauma elétrico* & $2(0,1)$ & $0(0,0)$ & $2(0,1)$ \\
\hline Trauma musculoesquelético isolado* & $151(2,5)$ & $2(1,8)$ & $153(2,5)$ \\
\hline
\end{tabular}

Fonte: Serviço de Atendimento Móvel de Urgência de Juiz de Fora, Minas Gerais.

'Trauma genérico: acidentes de menor frequência

Ao associar as variáveis sociodemográficas aos mecanismos de trauma, em relação à proporção entre os sexos, as mulheres foram as mais acometidas por quedas $(58,9 \%)$ e acidentes genéricos $(52,9 \%)$, enquanto os homens foram os mais expostos a violência interpessoal $(72,2 \%)$ e de trânsito $(58,7 \%)$. 
Tabela 3. Caracterização de atendimentos pré-hospitalar a idosos vítimas de trauma, 2014-2018 segundo o mecanismo de trauma $(\mathrm{n}=6040)$

\begin{tabular}{|c|c|c|c|c|c|c|}
\hline \multirow[b]{2}{*}{ Variável } & \multicolumn{4}{|c|}{ Tipo de trauma } & \multirow[b]{2}{*}{$\begin{array}{l}\text { Total } \\
\mathrm{n}(\%)\end{array}$} & \multirow[b]{2}{*}{$p$-value } \\
\hline & $\begin{array}{c}\text { Acidentes genéricos } \\
\mathrm{n}(\%)\end{array}$ & $\begin{array}{l}\text { Quedas } \\
\mathrm{n}(\%)\end{array}$ & $\begin{array}{c}\text { Violência interpessoal } \\
\mathrm{n}(\%)\end{array}$ & $\begin{array}{c}\text { Violência trânsito } \\
\text { n(\%) }\end{array}$ & & \\
\hline \multicolumn{7}{|l|}{ Sexo* ${ }^{*}$} \\
\hline Feminino & $157(52,9)$ & $2877(58,9)$ & $35(27,8)$ & $304(41,3)$ & $3373(55,8)$ & $<0,001$ \\
\hline Masculino & $140(47,1)$ & $2003(41,0)$ & $91(72,2)$ & $433(58,7)$ & $2667(44,2)$ & \\
\hline \multicolumn{7}{|c|}{ Faixa etária* (anos) } \\
\hline $60-69$ & $127(42,8)$ & $1381(28,3)$ & $78(61,9)$ & $374(50,8)$ & $1960(32,4)$ & $<0,001$ \\
\hline 70-79 & $81(27,3)$ & $1496(30,7)$ & $33(26,2)$ & $224(30,4)$ & $1834(30,4)$ & \\
\hline 80-89 & $66(22,2)$ & $1494(30,6)$ & $13(10,3)$ & $116(15,7)$ & $1689(28,0)$ & \\
\hline$\geq 90$ & $23(7,7)$ & $509(10,4)$ & $2(1,6)$ & $23(3,1)$ & $557(9,2)$ & \\
\hline \multicolumn{7}{|c|}{ Região geográfica* } \\
\hline Central & $104(35,0)$ & $1543(31,6)$ & $44(34,9)$ & $265(36,0)$ & $1956(32,4)$ & 0,002 \\
\hline Leste & $33(11,1)$ & $817(16,8)$ & $25(19,8)$ & $98(13,3)$ & $973(16,1)$ & \\
\hline Norte & $92(31,0)$ & $1407(28,8)$ & $31(24,6)$ & $221(30,0)$ & $1751(29,0)$ & \\
\hline Oeste & $13(4,4)$ & $278(5,7)$ & $5(4,0)$ & $31(4,2)$ & $327(5,4)$ & \\
\hline Sul & $47(15,8)$ & $727(14,9)$ & $16(12,7)$ & $96(13,0)$ & $886(14,7)$ & \\
\hline Rural & $8(2,7)$ & $108(2,2)$ & $5(4,0)$ & $26(3,5)$ & $147(2,4)$ & \\
\hline \multicolumn{7}{|l|}{ Óbito" } \\
\hline Não & $294(99,0)$ & $4797(98,3)$ & $122(96,8)$ & $714(96,9)$ & $5927(98,1)$ & 0,024 \\
\hline Sim & $3(1,0)$ & $83(1,7)$ & $4(3,2)$ & $23(3,1)$ & $113(1,9)$ & \\
\hline
\end{tabular}

Fonte: Serviço de Atendimento Móvel de Urgência de Juiz de Fora, Minas Gerais.

'Teste qui-quadrado.

Além disso, na comparação da proporção entre as faixas etárias, os idosos com 60 a 69 anos foram os que, com maior frequência, envolveram-se em violência no trânsito $(50,8 \%)$ e interpessoal $(6,9 \%)$, já os idosos entre 70 e 89 anos foram os mais expostos a quedas. A regiáo da cidade com mais registros de atendimento por quedas, violência interpessoal e no trânsito foi a área central (Tabela 3).

O óbito foi o desfecho mais frequente entre homens $(\mathrm{p}<0,001)$ e entre pessoas que sofreram quedas $(p=0,024)$. Não houve diferença na ocorrência de óbito entre as faixas etárias e entre as regióes da cidade (Tabela 4).

\section{Discussão}

A limitação deste estudo refere-se à não disponibilização de outras variáveis sociodemográficas no banco de dados eletrônico disponível. Além disso, os dados são de uma cidade de porte médio e podem não representar outras realidades. Por outro lado, os resultados da investigaçáo podem contribuir para a compreensão da ocorrência e dos mecanismos do trauma, o direcionamento de discussóes e açóes de enfermagem e saúde no âmbito da promoção e re-
Tabela 4. Associação entre variáveis independentes e a ocorrência de óbito $(n=6040)$

\begin{tabular}{|c|c|c|c|c|}
\hline \multirow{2}{*}{ Características } & \multicolumn{3}{|c|}{ Óbito } & \multirow{2}{*}{$p$-value* } \\
\hline & Não & Sim & Total & \\
\hline \multicolumn{5}{|l|}{ Sexo } \\
\hline Feminino & $3330(56,2)$ & $43(38,1)$ & $3373(55,8)$ & \\
\hline Masculino & $2597(43,8)$ & $70(61,9)$ & $2667(44,2)$ & $<0,001$ \\
\hline \multicolumn{5}{|l|}{ Faixa etária (anos) } \\
\hline $60-69$ & $1915(32,3)$ & $45(39,8)$ & $1960(32,4)$ & 0,135 \\
\hline $70-79$ & $1798(30,3)$ & $36(31,9)$ & $1834(30,4)$ & \\
\hline $80-89$ & $1662(28,0)$ & $27(23,9)$ & $1689(28,0)$ & \\
\hline$\geq 90$ & $552(9,3)$ & $5(4,4)$ & $557(9,2)$ & \\
\hline \multicolumn{5}{|l|}{ Região geográfica* } \\
\hline Central & $1919(32,4)$ & $37(32,7)$ & $1956(32,4)$ & 0,264 \\
\hline Leste & $953(16,1)$ & $20(17,7)$ & $973(16,1)$ & \\
\hline Norte & $1722(29,0)$ & $29(25,7)$ & $1751(29,0)$ & \\
\hline Oeste & $316(5,3)$ & $11(9,7)$ & $327(5,4)$ & \\
\hline Sul & $874(14,8)$ & $12(10,6)$ & $886(14,7)$ & \\
\hline Rural & $143(2,4)$ & $4(3,6)$ & $147(2,4)$ & \\
\hline \multicolumn{5}{|l|}{ Tipo de trauma* } \\
\hline Acidente genérico & $294(5,0)$ & $3(2,7)$ & $297(4,9)$ & 0,024 \\
\hline Quedas & $4797(80,9)$ & $83(73,5)$ & $4880(80,8)$ & \\
\hline Violência interpessoal & $122(2,1)$ & $4(3,5)$ & $126(2,1)$ & \\
\hline Violência trânsito & $714(12,0)$ & $23(20,3)$ & $737(12,2)$ & \\
\hline \multicolumn{5}{|l|}{ Ano* } \\
\hline 2014 & $775(13,1)$ & $23(20,4)$ & $798(13,2)$ & 0,014 \\
\hline 2015 & $1087(18,3)$ & $24(21,3)$ & $1111(18,4)$ & \\
\hline 2016 & $1275(21,5)$ & $20(17,7)$ & $1295(21,4)$ & \\
\hline 2017 & $1264(21,3)$ & $12(10,6)$ & $1276(21,2)$ & \\
\hline 2018 & $1526(25,8)$ & $34(30,0)$ & $1560(25,8)$ & \\
\hline
\end{tabular}

Fonte: Serviço de Atendimento Móvel de Urgência de Juiz de Fora, Minas Gerais. "Teste qui-quadrado. 
cuperação da saúde, além de políticas de prevenção ao trauma à população idosa.

Os resultados da investigação são compatíveis com a literatura nacional e internacional em relação ao trauma e a seus mecanismos de acometimento da pessoa idosa. ${ }^{(8,13-15)}$ As altas taxas de trauma e sua relação com a morbimortalidade dessa população evidenciam a necessidade de políticas públicas sólidas, planejamento social e urbano, além de açôes específicas de promoção e educação em saúde. ${ }^{(2,4)}$

$\mathrm{O}$ profissional de enfermagem, inserido nos diversas contextos de assistência, tem papel fundamental nas políticas e açóes de planejamento, promoção e educação em saúde ao idoso, de modo a atenuar o impacto dos fatores intrínsecos e extrínsecos na saúde dessa população. ${ }^{(16,17)}$ Além disso, são os profissionais que frequentemente prestam os primeiros atendimentos às vítimas, o que os torna protagonistas na prevenção de complicaçôes e redução de sequelas. ${ }^{(17)}$

No presente estudo, foi verificado predomínio do sexo feminino entre os idosos vítimas de trauma, com destaque para quedas. Esses dados corroboram achados na literatura, que ressaltam como possíveis fatores causadores diminuição da força muscular, osteoporose, restrição à vida doméstica, défices nas funçóes sensoriais que afetam o equilíbrio e a marcha na realização de tarefas, e a maior longevidade feminina. ${ }^{(18-20)}$

Pesquisas relacionam as fraturas ocasionadas por quedas em mulheres ao possível aumento da fragilidade osteomuscular iniciada no período pós-menopausa, devido à redução do estrógeno, além de maior exposição às atividades domésticas. ${ }^{(21,22)}$ Estudo longitudinal realizado na Inglaterra evidenciou outros fatores de risco para quedas entre as mulheres, como depressão, incontinência urinária, estado civil e baixa escolaridade. ${ }^{(23)}$

Cabe destacar que os mecanismos de trauma estão entre as principais causas de óbito em pessoas idosas, e as quedas representam até dois terços dos acidentes com idosos, tornando-se um dos principais previsores de morbimortalidade. ${ }^{(24,25)}$

Com o envelhecimento da população, o trauma torna-se um problema emergente e com progressão ao longo dos anos. Estudo desenvolvido em uma cidade de porte médio, na Região Nordeste do Brasil, constatou elevado número de atendimentos por trauma a faixas etárias mais avançadas, sendo que os idosos com idade acima de 80 anos eram do sexo feminino. ${ }^{(6)}$

A maioria dos idosos da presente pesquisa tinha faixa etária entre 60 e 79 anos, com média de 75 anos. Em estudos nacionais e internacionais, as médias de idade são variáveis, porém o trauma tende a ser mais frequente entre idosos jovens, ao passo que a morbimortalidade está diretamente relacionada à elevada idade e ao sexo..$^{(4,7,8,10)}$

Pesquisas relacionam a elevada idade a maiores chances de internação e mortalidade, considerando o declínio da capacidade funcional. ${ }^{(14,26,27)}$ No que diz respeito ao sexo, evidencia-se que a morbimortalidade é elevada entre os homens devido à tendência de se envolverem em atividades intensas e de risco. ${ }^{(8,23,28)}$ No presente estudo, o trauma, com destaque para as quedas, foi registrado de forma ampla em todas as faixas etárias. Em consonância com a literatura, a maioria dos homens da presente pesquisa apresentou trauma relacionado à violência interpessoal e a acidentes de trânsito. Além disso, ao se avaliar o desfecho dos atendimentos, verificou-se que os homens e as pessoas vítimas de quedas foram os mais vulneráveis ao óbito.

Entre o ano de 2014 e 2018, houve aumento progressivo e significativo dos registros de trauma envolvendo idosos, com destaque para região central da cidade. Acredita-se que esse achado tenha relação com a elevada concentração populacional, expondo um maior contingente de idosos a quedas, violência interpessoal e acidentes de trânsito, entre outros. ${ }^{(4,8)}$

Um estudo ${ }^{(4)}$ identificou que as capitais brasileiras concentraram elevadas taxas de óbitos e internaçóes provocadas por trauma em idosos, com aumento de $200 \%$ na taxa de mortalidade entre os anos de 1996 a 2012.

Assim, com o envelhecimento da população, torna-se necessária a ampla discussão sobre açóes de prevenção de doenças, promoção e recuperação da saúde, além de políticas e programas de gestão da segurança voltados aos idosos. ${ }^{(4,13,22)}$ 


\section{Conclusão}

Ao analisar registros e mecanismos de trauma em idosos socorridos por um Serviço de Atendimento Móvel de Urgência, verificou-se que a maioria dos envolvidos era do sexo feminino, havendo maior frequência de registro por quedas de altura ou da própria altura. Os mecanismos de trauma proporcionalmente mais frequentes no sexo masculino foram a violência interpessoal e de trânsito. Os idosos com faixa etária entre 60 e 69 foram mais expostos à violência no trânsito e à violência interpessoal; já os idosos entre 70 e 89 anos foram os mais expostos a quedas. A regiáo da cidade com mais registros de atendimento de trauma por quedas, violência interpessoal e de trânsito foi a área central. $\mathrm{O}$ óbito foi o desfecho mais frequente entre homens e entre pessoas que sofreram quedas.

\section{Agradecimentos}

Agradecemos à Universidade Federal de Juiz de Fora pelo apoio financeiro a esta pesquisa

\section{Colaborações}

Franck DBP, Alves KR e Carbogim FC foram responsáveis pela concepção do projeto, redação do artigo, análise e interpretação dos dados, revisão crítica relevante do conteúdo intelectual e contribuíram para a aprovação final da versão a ser publicada e são responsáveis pela totalidade do estudo, garantindo sua precisão e integridade. Costa YCN, Moreira TR, Sanhudo NF, Almeida GBS, Püschel VAA foram responsáveis pela revisão crítica relevante do conteúdo intelectual e contribuíram para a aprovação final da versão a ser publicada.

\section{Referências}

1. Instituto Brasileiro de Geografia e Estatística (IBGE). Projeção da população do Brasil e das Unidades da Federação [Internet]. Brasília (DF): IBGE; 2020 [citado 2020 Fev 29]. Disponível em: https://www. ibge.gov.br/apps/populacao/projecao/

2. Minayo MC. The imperative of caring for the dependent elderly person. Cien Saude Colet. 2019;24(1):247-52.
3. Cardona AM, Arango DC, Fernández DY, Martínez AA. Mortality in traffic accidents with older adults in Colombia. Rev Saude Publica. 2017;51(0):21.

4. Abreu DR, Novaes ES, Oliveira RR, Mathias TA, Marcon SS. Fall-related admission and mortality in older adults in Brazil: trend analysis. Cien Saude Colet. 2018;23(4):1131-41.

5. Porto Gautério D, Zortea B, Costa Santos SS, da Silva Tarouco B, Lopes MJ, João Fonseca C. Risk Factors for new accidental falls in elderly patients at traumatology ambulatory center. Invest Educ Enferm. 2015;33(1):35-43.

6. Franklin $T A$, Santos $H C$, Santos Júnior JA, Vilela $A B$. Caracterização do atendimento de um serviço pré-hospitalar a idosos envolvidos em queda. Rev Pesqui Cuid Fundam Online 2018;10(1):62-67.

7. Sawa J, Green RS, Thoma B, Erdogan M, Davis PJ. Risk factors for adverse outcomes in older adults with blunt chest trauma: A systematic review. CJEM. 2018;20(4):614-22.

8. Santos AM, Rodrigues RA, Diniz MA. Trauma by traffic accident in elderly people: risk factors and consequences. Texto Contexto Enferm. 2017;26(2):e4220015

9. TabNet Win32 3.0: Morbidade Hospitalar do SUS por Causas Externas - por local de internação - Brasil [Internet]. Brasília (DF):DATASUS; 2013 [citado 2019 Nov 14]. Disponível em: http://tabnet.datasus.gov. br/cgi/tabcgi.exe?sih/cnv/fiuf.def

10. Naeem Z, McCormack JE, Huang EC, Vosswinkel JA, Shapiro MJ, Zarlasht $F$, et al. Impact of Type and Number of Complications on Mortality in Admitted Elderly Blunt Trauma Patients. J Surg Res. 2019;241:78-86.

11. Lentsck MH, Sato AP, Mathias TA. Epidemiological overview - 18 years of ICU hospitalization due to trauma in Brazil. Rev Saude Publica. 2019;53:83.

12. Brasil. Ministério da Saúde. Secretaria de Ciência, Tecnologia e Insumos Estratégicos. Departamento de Ciência e Tecnologia. Agenda de Prioridades de Pesquisa do Ministério da Saúde - APPMS [Internet]. Brasília (DF): Ministério da Saúde; 2018. [citado 2020 Mar 12]. Disponível em: http://bvsms.saude.gov.br/bvs/publicacoes/agenda_ prioridades_pesquisa_ms.pdf

13. Fernandes $\mathrm{CM}$, Boing $\mathrm{AC}$. Pedestrian mortality in road traffic accidents in Brazil: time trend analysis, 1996-2015. Epidemiol Serv Saude. 2019;28(1):e2018079.

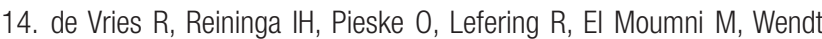
K. Injury mechanisms, patterns and outcomes of older polytrauma patients-An analysis of the Dutch Trauma Registry. PLoS One. 2018;13(1):e0190587.

15. Beck B, Cameron P, Lowthian J, Fitzgerald M, Judson R, Gabbe BJ. Major trauma in older persons. BJS Open. 2018;2(5):310-8.

16. Bérubé $M$, Pasquotti $T$, Klassen $B$, Brisson $A$, Tze $N$, Moore $L$. Implementation of the best practice guidelines on geriatric trauma care: a Canadian perspective. Age Ageing. 2020;49(2):227-32.

17. Degani GC, Mendes KD, Storti LB, Marques S. Advanced mobile prehospital nursing care for elderly people post-trauma: integrative review. Rev Bras Enferm. 2019;72 Suppl 2:274-83.

18. Leitão SM, Oliveira SC, Rolim LR, Carvalho RP, Coelho Filho JM, Peixoto Júnior AA. Epidemiologia das quedas entre idosos no Brasil: uma revisão integrativa de literatura. Geriatr Gerontol Aging. 2018;12(3):172-9.

19. Wong TH, Wong YJ, Lau ZY, Nadkarni N, Lim GH, Seow DC, et al. Not All Falls Are Equal: Risk Factors for Unplanned Readmission in Older Patients After Moderate and Severe Injury-A National Cohort Study. J Am Med Dir Assoc. 2019;20(2):201-207.e3.

20. Welmer AK, Rizzuto D, Laukka EJ, Johnell K, Fratiglioni L. Cognitive and Physical Function in Relation to the Risk of Injurious Falls in Older Adults: A Population-Based Study. J Gerontol A Biol Sci Med Sci. 2017;72(5):669-75. 
21. Xavier PF, Trindade AP. Avaliação do risco de queda e equilíbrio em mulheres no climatério. Rev Kairós Gerontol. 2018;21(2):15570.

22. Manlapaz DG, Sole G, Jayakaran P, Chapple CM. Risk Factors for Falls in Adults with Knee Osteoarthritis: A Systematic Review. PM R. 2019;11(7):745-57.

23. Gale CR, Westbury LD, Cooper C, Dennison EM. Risk factors for incident falls in older men and women: the English longitudinal study of ageing. BMC Geriatr. 2018;18(1):117.

24. Vieira LS, Gomes AP, Bierhals I0, Farías-Antúnez S, Ribeiro CG, Miranda $\mathrm{VI}$, et al. Falls among older adults in the South of Brazil: prevalence and determinants. Rev Saude Publica. 2018;52:22.
25. Carneiro JA, Ramos GC, Barbosa AT, Vieira ÉD, Silva JS, Caldeira AP. Falls among the non-institutionalized elderly in northern Minas Gerais, Brazil: prevalence and associated factors. Rev Bras Geriatr Gerontol. 2016;19(4):613-25.

26. Chang JC, Yuan ZH, Lee IH, Hsu TF, How CK, Yen DH. Pattern of nontrauma emergency department resource utilization in older adults: An 8-year experience in Taiwan. J Chin Med Assoc. 2018;81(6):552-8.

27. Oliveira AC, Rocha DM, Bezerra SM, Andrade EM, Santos AM, Nogueira LT. Quality of life of people with chronic wounds. Acta Paul Enferm. 2019;32(2):194-201.

28. Zanette GZ, Waltrick RS, Monte MB. Epidemiological profile of thoracic trauma in a reference hospital of Foz do Rio Itajai. Rev Col Bras Cir. 2019;46(2):e2121. 